\title{
The Design of the Shock Mitigation System of Large Cone Crusher
}

\author{
Gao Jingjun, Li Zhixing, Shan Changzheng, and Gao Wei
}

\begin{abstract}
This paper's study is according to the large cone crusher of Ansteel Mining. It elaborates the characteristics and design requirements of composite spring, a part of the shock mitigation system of large cone crusher. The paper details the Shock absorption principle of composite spring of large cone crusher. We will calculate the main parameters of the composite spring shock absorption pad to ensure that it's rigidity meet the requirements of working conditions. So, the study can provide a theoretical basis for the further research and development of the crusher.
\end{abstract}

Index Terms-Crusher, composite spring, shock absorption pad, design.

\section{INTRODUCTION}

Ansteel Mining is Ansteel Group Corporation's capitalization subsidiary company. It Is China's control of iron ore resources, the largest maximum yield, with the operation mode of process technology, advanced low cost in exploration, mining, ore dressing, sintering, pellet production, mining technology research and development, design, engineering and technical output as a whole, has a complete industrial chain of metallurgical mine hill leading enterprises. Ansteel Group Corporation is Chinese metallurgy mine's flagship enterprise. The company started to import large quantities of large cone crusher since 90's of the last century. Medium crushers are largely single cylinder hydraulic cone crushers, while the fine crushers are mainly multi cylinder hydraulic ones. So, the company has accumulated a lot of maintenance and use experience of crushers. Cone crusher, the key equipment of beneficiation plants, locates in the final stage of the ore crushing production line. In recent years, users and manufacturers all have a strong interest in cone crushers due to the demand and changes in the market. They introduce foreign advanced cone crusher to understand and assimilate the new technology, and then they will carry on research and innovation. Composite spring, the shock absorber part of crushers, is the most widely used type. Composite spring, rubber metal spiral spring, is made by composite vulcanization of metal spiral spring which is wrapped around a layer of rubber material.

Manuscript received November 17, 2014; revised April 10, 2015.

Gao Jingjun is with the Ansteel Mining, China (e-mail: musuiye@sina.cn).

Li Zhixing is with the Inner Mongolia University of Science and Technology, China (e-mail: \{onyxlzx, changzheng815\}@126.com).

Shan Changzheng is with the University of Science and Technology Beijing, China (e-mail: \{onyxlzx, changzheng815\}@126.com).

Gao Wei is with the University of Science and Technology Beijing, China (e-mail: 1124048511@qq.com).

\section{FeAtures AND Design ReQuiREMENTS of Composite SPRING}

\section{A. Composite Spring's Characteristics of Mitigation System of Crusher}

The characteristics of composite spring mitigation system are as follow [1]:

1) The shock absorption capacity of rubber is much larger than the metal because it can absorb and convert vibration into heat. Rubber is suitable for high frequency vibration, because it has damping characteristic.

2) It is not sensitive to water and dust. It will not produce sudden destruction in use. So, it is safe and reliable. And then it also has low cost and long enough service life. Rubber spring mitigation system has many advantages like: the simple structure, convenient use and maintenance, almost no repair, no wear, no rust, no need of lubricating oil and so on. Because composite spring mitigation system has the above features, it is widely used in crusher.

3) The shape of the composite spring mitigation system can be chosen arbitrarily. The three direction's rigidity, upper and lower, left and right, before and after, can obtain through reasonable design of the structure of damping element. It can simplify the mitigation system greatly.

4) Rubber and metal can be firmly combined.

5) Composite spring mitigation system has deformation characteristics of three directions, so larger errors are allowed when it is assembled.

Composite spring mitigation system applied in the large cone crusher is influenced by vibration. Then its main function is to bear compressive stress, shear stress and torsion moment, or the composite function of two or more than two kinds of stress at the same time. Consequently composite spring mitigation system plays an important role in the crusher.

\section{B. Composite Spring's Design Requirements of Mitigation System of Crusher}

Large cone crusher mitigation system can choose suitable materials according to screening materials [2]. Shock mitigation system can be classified according to the material as follows: rubber spring, composite spring, hardware spring, air spring, cork, blankets and so on. Composite spring can cancel the damper. The equipment is more stable when it is passing through the resonance region, and the time is shorter. It can ease the impact; reduce noise, corrosion and dust. Even if the metal spring breaks, equipment accidents will not occur. Every composite spring of the crusher bears 3 tons. And the size is length*width*height $=310 \mathrm{~mm} * 270 \mathrm{~mm} * 120 \mathrm{~mm}$. 
Design requirements of crusher:

1) Select appropriate rubber according to the working conditions and surroundings;

2) Be caution of the bonding degree of rubber and metal to avoid the stress concentration at bonding surface;

3) When it comes to the shear deformation of the vibration isolation system, we usually give appropriate pre compression in the direction perpendicular to the shear direction, in order to improve the life, and then compression stiffness hardens while the shear direction stiffness of soft;

4) Rubber shock pad should not work in long-term tensile state;

5) Damping will consume energy which will be converted into heat. Rubber is a poor conductor of heat. To prevent excessive temperature rise which will affect the performance of the rubber vibration isolator the following matters need to pay attention: rubber isolator should not be too large, take easy heat dissipation measures in the structure, or select natural rubber material that produce less heat Rubber spring is called shock absorber. Because rubber vibration isolator can convert part of energy into heat, which will reduce the vibration energy and achieve the purpose of damping.

\section{THE WORKING PRINCIPLE OF COMPOSITE SPRING}

Rubber shock pad of crusher generally use natural rubber and chloroprene rubber. When it has requirements of oil resistant, nitrile rubber is used. When it has the requirements of high and low temperature resistance, silicon rubber is adopted. When it has the requirements of high damping, butyl rubber is used. Manufacturing process of most rubber shock pad products is similar to model products, while rubber air spring is similar to tire. The rubber has high elastic state and high viscosity state. The elasticity of the rubber is produced by changes in the molecular conformation of curl. Rubber molecular interaction will hinder the movement of molecular chain and show the viscous characteristics, which will lead stress and strain to a state of imbalance. Curly long chain molecular structure of rubber and the weak secondary force between molecules make rubber material a unique viscoelastic properties exhibition. So it has good shock absorption, sound insulation and damping property. Because it has lag, damping and can be reversible with large deformation, so rubber parts are widely used in isolating vibration and shock absorption. The hysteresis and the internal friction characteristics of rubber are usually expressed as the loss factor. The greater loss factor is, the more significant rubber damping and heat is and the more obvious damping effect is [3]-[5].

Rubber material loss factor is not only related to the structure of rubber but also the temperature and frequency. Under normal temperature, the loss factor natural rubber (NR) and butadiene rubber (BR) is smaller. The loss factor of styrene butadiene rubber (SBR), chloroprene rubber (CR), ethylene propylene rubber (EPR), polyurethane rubber (PU) and silicon rubber in the middle. Butyl rubber (HR) and nitrile rubber (NBR) have the biggest loss factor.

Rubber material used in Shock absorption is generally divided into 5 kinds: That is NR, SBR, BR is the ordinary rubber material; NBR for the oil resistance of vulcanized rubber; $\mathrm{CR}$ is used for weather resistance of vulcanized rubber; IIR for high damping vulcanized rubber; EPR is used for heat resistance of vulcanized rubber. Although the loss factor of NR is small, it has the best comprehensive properties like: excellent flexibility, fatigue resistance, processing properties, low heat, and little creep, good performance in metal bonding, cold resistance, and electrical insulation properties. So NR is widely used in shock absorber. When it has requirements of low temperature or weather resistant performance, we can use NR with $\mathrm{BR}$ or $\mathrm{CR}$ at the same time or have a mixed use to modify. Shock absorber has better durability. Shock absorber that made by NR BR and metal salt that have more than four carbon atoms containing organic acid with $-\mathrm{OH}$ groups have a good durability. Under the condition of $70^{\circ} \mathrm{C} \times 22 \mathrm{~h}$ and $40^{\circ} \mathrm{C} \times 148 \mathrm{~h}$, compression permanent deformation is $17.0 \%$ and $11.7 \%$.

\section{Parameters Calculation of the Composite Spring SHOCK ABSORPTION PAD}

Hardness of rubber is the main parameter that determines their performance. The greater the hardness of rubber, the greater the strength and the worse of elastic and damping performance is. Take various factors into consideration, the best hardness value of rubber shock pad is $\mathrm{HA}=40 \sim 90$.

When elasto viscous rubber material is subjected to impact, part of the kinetic energy is converted into heat energy which will be consumed. So it has a good function of noise and vibration reduction. Crusher vibration transmitted to the foundation will cause vibration and noise. The shock pad is arranged between the crusher and the foundation, and it will reduce the vibration transmission. In general, a shock pad has a symmetric distribution according to the crusher center, and has effect of support, shock absorption and noise reduction. Under the excitations of rusher vibration, the shock pad will produce the corresponding natural frequency of vibration:

$$
f_{n}=\frac{1}{2 \pi} \sqrt{\frac{K^{\prime} g}{G}}
$$

In the formula:

$f_{n}$ — the natural frequency of the shock absorption pad, $\mathrm{Hz}$;

$K^{\prime}$ - the dynamic stiffness of the shock absorption pad, N.mm ${ }^{-1}$;

$g-$ Standard acceleration of gravity, $9800 \mathrm{~mm} . \mathrm{s}^{-2}$;

$G-$ Crusher gravity, N.

The vibration transmission rate of the shock pad $\left(\eta_{A}\right)$, The input vibration transmission rate decides the damping ratio $(\eta)$, express damping capacity of shock pad, $\eta=1-\eta_{A}$. The smaller $\eta_{A}$ is, the better the damping effect of shock pad [6]. The formula between $\eta_{A}$ and the loss factor of damping pad $(\tan \delta)$, vibration frequency of crusher $(f)$, specific value $\left(f_{n}\right)$ is: 


$$
\eta_{A}=\sqrt{\frac{1+(v \tan \delta)^{2}}{\left(v^{2}-1\right)^{2}+(v \tan \delta)^{2}}}
$$

From the above formula, we can see there is a nonlinear relationship between $\eta_{A}$ and $v$. With the increase of $v, \eta_{A}$ increases rapidly at the beginning and reached maximum value at $v=1$. At this point the system resonance, resulting in strong vibration of foundation of crusher. Thus it causes the crusher work abnormally and fatigue damage of shock pad. Then $\eta_{A}$ decreases, and the different $\tan \delta$ curves were handed over to the $v=\sqrt{2}$. When $v \leq \sqrt{2}, \eta_{A} \geq 1$, shock pad cannot play a damping effect. When $v>\sqrt{2}, \eta_{A}<1$, the shock pad play a damping effect. The smaller the $\tan \delta$, the greater the $v$, the greater the magnitude of the decrease of $\eta_{A}$, the better the damping effect. So when we design damping pad, we should choose low damping rubber material and pay attention to choice of the value at the same time. The $f$ of crusher is fixed, so we adjust the $f_{n}$ of damping pad to control $v$. But $v$ cannot be too large, otherwise the $f_{n}$ of the shock pad is too low, the $K^{\prime}$ of shock pad is too small accordingly. And then to achieve the same damping effect, the size of the damping pad is too large. We can that the general selection of $v$ is $2.4-4.5$ by experiment.

When $v>\sqrt{2}$, for low damping rubber material $(\tan \delta \approx 0$ ),

$$
\eta_{A}=\sqrt{\frac{1+(v \tan \delta)^{2}}{\left(v^{2}-1\right)^{2}+(v \tan \delta)^{2}}}
$$

can be simplified to:

$$
\eta_{A}=1 /\left(v^{2}-1\right)
$$

In the active damping, rubber shock pad will bear the gravity of crusher. So, compression permanent deformation of rubber material in use is small. For the convenience of calculation, assuming the stress-strain relationship is linear relationship. So we can use the effective modulus of compression (function of rubber material properties and geometry of the shock pad) to evaluate the characteristics of damping pad [7].

$$
K_{s}=S E_{c} / h
$$

In the formula:

$K_{s} \longrightarrow$ the static stiffness of the shock pad, N.mm ${ }^{-1}$;

$E_{c}$ the effective modulus of compression of the shock pad, $\mathrm{MPa}$;

$S$ _the effective loading area of the shock pad, $\mathrm{mm} 2$;

$h$ the height of the shock pad, mm.

The $K_{s}$ of the rubber item is smaller than $K^{\prime}$. The difference increases with the rubber material damping value increasing. $K^{\prime} / K_{S}$ is 1.2-2 in general. Geometry of shock pad has tremendous impact on modulus. The formula between $E_{c}$ and form factor (load size and loading area ratio of shock pad) is:

$$
E_{c}=E_{0}\left(1+2 k C^{2}\right)
$$

In the formula:

$E_{0}$ - the young's modulus of rubber material;

$C$ _ the form factor of damping pad;

$K$ Factor correction of material characteristics (Deviation between correction calculation value and the experimental values), its value is generally $0.5-1$.

For low damping rubber material, we assume that the limit of static stress $(\sigma)$ is $0.069 \mathrm{MPa}$. And then we can find the minimal loading area of the shock pad:

$$
S=Q / \sigma
$$

In the formula:

$Q$ - Static load of shock pad, N;

$\sigma$ - the static stress.

In order to avoid stress concentration, shock pad should have the appropriate structure [8]. The length and width of crusher spring were $310 \times 270$, so the minimum $S$ value for each shock pad is $83700 \mathrm{~mm}^{2}$. Shock pad that $\eta$ is 0.8 can make the crusher vibration and noise greatly reduced and meet the safety and usage requirements. So the $\eta_{A}$ of shock pad is 0.2 . We can find $f_{n}$ is $20 \mathrm{~Hz}$ by $\eta_{A}=1 /\left(v^{2}-1\right)$ and $f_{n}=\frac{1}{2 \pi} \sqrt{\frac{K^{\prime} g}{G}}$. The $K^{\prime}$ of damping system is $23700 \mathrm{~N} \cdot \mathrm{mm}^{-1}$. Select a smaller value $K^{\prime} / K_{S}$ of damping system. And it is 1.4. So $K_{s}$ of damping system is $5912 \mathrm{~N} . \mathrm{mm}^{-1}$. The $K_{s}$ of every shock pad is $1478 \mathrm{~N} . \mathrm{mm}^{-1}$.

We can select the $E_{c}$ of shock pad as 2.1Mpa.Then we can find $h$ is $119 \mathrm{~mm}$ by $K_{s}=S E_{c} / h$.

\section{CONCLUSION}

Through the above analysis we can see every shock pad whose $K_{s}$ is 1478 N.mm ${ }^{-1}$ and rectangular rubber shock pad whose $h$ is $119 \mathrm{~mm}$ can reduce the vibration and noise of the crusher effectively. So, the study can provide a theoretical basis for the further research and development of the crusher.

\section{REFERENCES}

[1] W. Wang, Q. H. Zhao, and H. Zhang, "Dynamic damper parameter optimization analysis," Journal of Vibration and Shock, 2012.

[2] H. H. Wang and J. Z. Wu, "Shaker harmful coupled vibration suppression countermeasure,” Shenyang Gold College, vol. 30, no. 3 pp. 287-291, 2004.

[3] W. L. Wang, T. T. Wang, J. J. Su et al., “Assessment of damage in mountain tunnels due to the Taiwan Chi-Chi Earthquake,” Tunneling and Underground Space Technology, vol. 29, no. 16, pp. 133-150, 2001. 
[4] G. J. Wang, Q. D. Yan, and X. F. Meng, "Automobile shock absorber bearing fatigue cracking cause analysis," Journal of Agricultural Equipment and Vehicle Engineering, vol. 25, no. 5, 2006.

[5] L. Z. Ma, "Automotive hydraulic shock absorbers to optimize the design parameters," Automotive Technology, vol. 10, no. 3, pp. 24-28, 2004.

[6] B. W. Wang and J. Y. Ge, "Giant magnetostrictive material and its application,” Shenyang University of Technology, vol. 20, no. 3, pp. 62-68, 1998.

[7] Z. H. Chen and Y. J. Huang, "Locomotives with an elastic element and development trend," Chinese Rubber, vol. 16, no. 19, pp. 5-26, 2000.

[8] Y. N. Zheng and L. D. Yang, Underground Seismic, Shanghai: Tongji University Press, 2005.

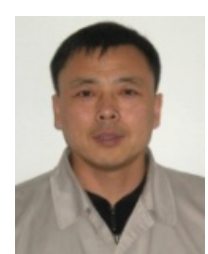

Gao Jingjun is with the Ansteel Mining, China. He is senior engineer, postgraduate student, major in mechanical design and theory

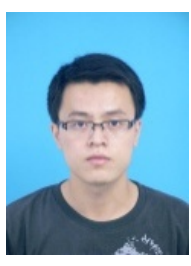

Li Zhixing is with the Inner Mongolia University of Science and Technology, Ph.D Candidate, major in dynamics of vehicle .

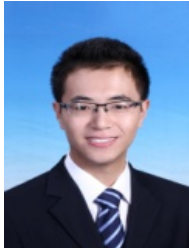

Shan Changzheng is with the University of Science and Technology Beijing, China. He is a postgraduate student, major in Mechanical design and theory.

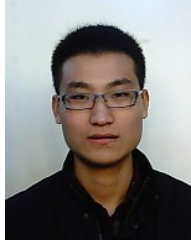

Gao Wei is with the University of Science and Technology Beijing, China. He is a postgraduate student, major in mechanical design and theory. 